\title{
ATOMIC DATA NEEDED FOR FUV ASTRONOMY WITH HUT AND FUSE
}

\author{
JEFFREY L. LINSKY ${ }^{1}$ \\ Joint Institute for Laboratory Astrophysics \\ NIST and University of Colorado \\ Boulder, Colorado 80309-0440 \\ USA
}

\begin{abstract}
The important atomic and molecular data needed by astrophysicists who analyze far ultraviolet spectra include accurate wavelengths, ocsillator strengths, molecular data for six molecules, and especially electron collisional exciatation cross sections.
\end{abstract}

I will summarize the types of atomic and molecular data that astrophysicists need for analyzing spectra obtained in the far ultraviolet $(912-1250 \AA)$ and extreme ultraviolet $(100-912 \AA)$. I separate these two spectral regions at the Lyman edge, because near $912 \AA$ the instrumental techniques and the temperatures at which the important diagnostics are formed change character appreciably.

\section{Instrumental Characteristics of HUT and FUSE}

IUE and the HST spectrographs, the FOS and the GHRS, retain some sensitivity down to $\lambda \approx 1150 \AA$. Until recently, our only glimpses of spectra of astronomical sources other than the Sun came from the Copernicus satellite and a few sounding rocket experiments. HUT, the Hopkins Ultraviolet Telescope, has provided high signal/noise spectra of many types of sources during the 1990 ASTRO-1 mission. A reflight in 1993 is planned. HUT contains a $90 \mathrm{~cm}$ telescope feeding a Prime Focus Spectrograph with $3.0 \AA$ spectral resolution in the $425-1850 \AA$ range.

After its launch in the year 2000, FUSE, the Far Ultraviolet Spectrograph Explorer, will provide very sensitive high-resolution spectra. Present plans call for a resolution of $\lambda / \Delta \lambda \approx 30,000$ in the $910-1250 \AA$ region to be obtained with a $1.6 \mathrm{~m}$ Rowland Spectrograph with about $50 \mathrm{~cm}^{2}$ effective area. There will also be a "planetary" channel covering the $400-1600 \AA$ range with a resolution of 1,000 . The FUV contains such important diagnostics as the Lyman lines of $H$ and $D$, the molecules $\mathrm{H}_{2}$ and $\mathrm{HD}$, the resonance lines of $\mathrm{C}$ III and $\mathrm{O}$ VI, and forbidden lines of coronal ions. In the EUV, FUSE will have a glancing-incidence spectrograph covering the $100-350 \AA$ range with resolutions up to 2,500 . The EUV contains many bright lines of highly-ionized species like Fe XI-XXIV diagnostic of $10^{6}-10^{7} \mathrm{~K}$ plasmas.

${ }^{1}$ Staff Member, Quantum Physics Division, National Institute of Standards and Technology 


\section{Spectroscopic Needs}

The analysis of FUV and EUV spectra from HUT and FUSE will require new measurements in the laboratory along the following lines:

- Accurate wavelengths and energy levels. Accurate absolute wavelengths are needed, for example, to measure downflow and wind expansion velocities in stars and to Doppler image bright or dark or chemically peculiar regions on stellar surfaces. Resonance, intersystem, and forbidden lines of moderately jonized $\mathrm{C}, \mathrm{N}$, and $\mathrm{O}$ are most important. Since line center wavelengths can be measured to $\geq 10 \lambda \Delta \lambda$, laboratory measurements must be at least this good.

- Oscillator strengths. Abundance analyses for stars and the interstellar medium are often limited by the accuracy of oscillator strengths of C I+II, N I+II, O I+II, Ne II, Mg I, P II, Si II, and Fe I+II. Of particular inportance to interstellar work are the C II] $2325 \AA$ and Si II] $2335 \AA$ multiplets. For higher stages of ionization, a few accurate laboratory measurements are needed to test the accuracy of theoretical predictions.

- Molecular data in the FUV. One needs accurate wavelengths, photoionization cross sections, and oscillator strengths for six important molecules $-\mathrm{H}_{2}$, $\mathrm{HD}, \mathrm{CO}, \mathrm{N}_{2}, \mathrm{C}_{2}$, and $\mathrm{OH}$. Oscillator strengths are in good shape for the first three molecules and the $\mathrm{H}_{2}$ fluorescence processes are probably understood.

\section{Electron Collisional Excitation Needs}

The single most important class of atomic data needed for the analysis of FUV and EUV spectra is electron collisional excitation cross sections. These data are needed for estimating electron densities and understanding ionization equilibria. I encourage both experimental and theoretical work in the following areas:

- Intersystem transitions. Line flux ratios within intersystem multiplets or between intersystem and permitted lines are commonly used as density diagnostics when the collisional excitation rates for the intersystem transitions are known accurately. In the FUV, density-sensitive lines are present in C III, N III, Ne V+VI, Si IV, and S III+IV. In the EUV important species are O VI, Mg VII+VIII, Si IX, S XI, Ca XV-XVII, and Fe IX-XV and XXI.

- Highly ionized species. More accurate rates are needed for the Li-isoelectronic sequence (e.g. Mg X, Si XII,...) and Fe IX-XIX, including cascades.

- S II-IV. HUT spectra of the Io Torus show many of these lines.

- Low stages of ionization of abundant species. The ions of interest include $\mathrm{C} \mathrm{I}+\mathrm{II}, \mathrm{N} \mathrm{I}+\mathrm{II}$, and $\mathrm{O} \mathrm{I}+\mathrm{II}$.

- Dielectrionic recombination cross sections for many ions. These data are needed for ionization equilibria calculations of photoionized gases, especially at low temperatures $\left(\sim 10^{4} \mathrm{~K}\right)$ where resonances are most important. 